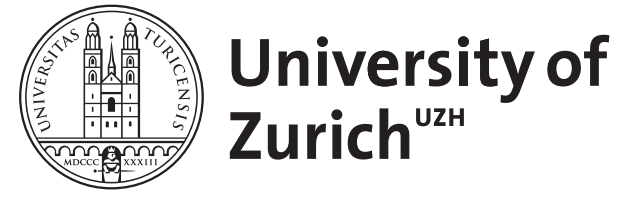

Zurich Open Repository and Archive

University of Zurich

University Library

Strickhofstrasse 39

CH-8057 Zurich

www.zora.uzh.ch

Year: 2020

\title{
Avoiding Dual Graft Loss in Simultaneous Liver Retransplantation and Primary Kidney Transplantation
}

Petrowsky, Henrik

DOI: https://doi.org/10.1097/tp.0000000000003036

Posted at the Zurich Open Repository and Archive, University of Zurich ZORA URL: https://doi.org/10.5167/uzh-183754

Journal Article

Accepted Version

Originally published at:

Petrowsky, Henrik (2020). Avoiding Dual Graft Loss in Simultaneous Liver Retransplantation and Primary Kidney Transplantation. Transplantation, 104(7):1328-1329.

DOI: https://doi.org/10.1097/tp.0000000000003036 


\section{Transplantation Publish Ahead of Print}

\section{DOI: 10.1097/TP.0000000000003036}

Avoiding dual graft loss in simultaneous liver retransplantation and primary

\section{kidney transplantation}

Henrik Petrowsky, MD, FACS

Swiss HPB and Transplantation Center Zurich, Department of Surgery and Transplantation, University Hospital Zurich, Zurich, Switzerland

Short running title: Simultaneous liver retransplantation and kidney transplantation

Conflicts of Interest: The author has no conflicts of interest to declare.

Comment on: Cauchy F, Vibert E, Cherqui D, et al. Liver retransplantation associated with kidney transplantation for end stage liver graft disease and renal insufficiency: a morbid procedure on a unique subgroup of patients. Transplantation. IN PRESS.

Address of Correspondence: Henrik Petrowsky, MD, FEBS (HPB), FACS

Swiss HPB and Transplantation Center Zurich, Department of Surgery and Transplantation, University Hospital Zurich, Raemistrasse 100, 8091 Zurich, Switzerland. Telephone: +4144 255 3000, Email: henrik.petrowsky@usz.ch 


\section{Abbreviations}

D-MELD Donor age $\times$ MELD score

ESLD End-stage liver disease

MELD Model of end-stage liver disease

ReLT-KT Simultaneous liver retransplantation and kidney transplantation

SLKT Simultaneous liver and kidney transplantation

UCLA University of California Los Angeles

UNOS United Network of Organ Sharing 
Simultaneous liver and kidney transplantation (SLKT) is an increasingly performed procedure in patients with end-stage liver disease (ESLD) and renal failure. ${ }^{1}$ According to a recent report, more than 700 SLKT were performed in the United States in 2016 representing $9.3 \%$ of all annual liver transplants. ${ }^{2}$ This proportion has been continuously growing during the past decade and accounted for $2 \%$ only in the year 2000. In adult SLKT, chronic renal disease and sustained acute kidney injury are the 2 dominant causes of renal failure. In critically ill liver transplant candidates with underlying chronic kidney disease, renal failure might be often driven by acute-on-chronic conditions.

Cauchy and colleagues reported on a unique cohort of patients undergoing simultaneous liver retransplantation and kidney transplantation (ReLT-KT). ${ }^{3}$ Although several studies reported outcomes of SLKT, none of those focused exclusively on the scenario of liver retransplantation. The authors of 2 French liver transplant units analyzed the outcome of 45 recipients who underwent ReLT-KT during the period from 1994 to 2012. In parallel to the trends of growing SLKT procedures in the United States, the French study observed similar increased rates of ReLT-KT over time. In this study, the most common indications for liver retransplantation were recurrent liver disease and cholangitis, while chronic renal failure due to calcineurin inhibitor toxicity was the leading indication for simultaneous kidney transplantation. Although the authors differentiated between 4 clinical scenarios, the decision making for ReLT-KT was done on a case-by-case basis and did not rely on established guidelines for simultaneous kidney transplantation. This fact highlights the long lasting problem of lacking established criteria when to proceed with simultaneous kidney transplantation. ${ }^{4}$ Even in the United States, there were no formal criteria for SLKT before August 2017 and centers had to request simultaneous kidney transplantation according to their need and clinical judgement. Meanwhile, UNOS and other nonprofit transplant organizations have recently implemented eligibility criteria for SLKT. ${ }^{2}$ However, we have to 
keep in mind that the study period of the French cohort closed in 2012 when worldwide no formal criteria for SLKT existed. Therefore, there is an urgent need for outcome research of contemporary SLKT cohorts, which follow implemented eligibility criteria for additional kidney transplantation in primary liver transplantation or retransplantation settings.

The central finding of the French study was the high early mortality rate of $27 \%$ among recipients undergoing ReLT-KT. ${ }^{3}$ The authors identified D-MELD (donor age $\times$ MELD score) as only independent factor associated with overall survival. This finding indicates that donor age and MELD score significantly determine early outcome after ReLTKT. Under this perspective, donor age might represent a certain surrogate marker for donor organ quality for both liver and kidney while MELD score reflects the hepatic and renal medical acuity of the recipient. In other words, critically ill recipients undergoing ReLT-KT with organs from donors with advanced age do poorly and have a high risk to experience futile outcome. Probably everybody would agree with the authors that this finding has important clinical implications for appropriate selection of ReLT-KT candidates. The pattern of risk factors of the French study adds to the findings of a recently published study from the University of California Los Angeles (UCLA) in an even sicker recipient population. ${ }^{5}$ In this study, 145 patients underwent SLKT of whom 35 had ReLT-KT with a median MELD score of 35 compared to a MELD score of 23 in the French study. However, both studies differed significantly in their composition of underlying causes for end-stage renal disease. The study by Cauchy and colleagues had an almost-zero rate of acute kidney injury ( $2 \%$ versus $43 \%$ ) but an overwhelming rate of chronic kidney disease (98 versus 57\%) compared to the study from UCLA. Regardless of these differences, retransplantation, MELD score, and kidney donor risk index with age as component variable were independently associated with renal allograft futility and mirrored in some extent the risk profile of the French study. However, we have to keep in mind that early mortality is not only an unfortunate event for the recipient 
but it also implies a loss of 2 donor organs with significant impact on patients on the liver and kidney waiting list. ${ }^{6}$ Therefore, the study significantly contributes to the important body of evidence that elucidates the question of whether to proceed or defer simultaneous kidney transplantation or even ReLT-KT.

Interestingly, the subgroup analysis of the French study revealed that the highest 90day mortality of approximately 50\% was observed in patients with dominant and/or severe liver impairment while recipients with dominant kidney and/or moderate liver impairment had zero mortality. ${ }^{3}$ The authors argue that these findings imply that ReLT-KT needs to be considered earlier before severe liver impairment occurs (Figure 1) and that high acuity recipients should receive optimal organs. Although these scenarios are desirable, reality is often different especially for transplant centers facing extreme recipients (MELD 35+). Therefore, delayed or deferred kidney transplantation after liver transplantation might be a reasonable option in very sick recipients requiring ReLT-KT. The principle behind this strategy is to avoid simultaneous kidney implantation in a hostile and unstable environment when renal allograft futility is likely. In a recent study, 63 of 130 combined liver and kidney transplantation had delayed kidney implantation by average of 50 hours of cold ischemia while maintained on machine perfusion. ${ }^{7}$ The authors of this study reported improved survival for patients undergoing delayed versus simultaneous kidney transplantation. If delayed kidney implantation is not an option due to prolonged critical illness after liver transplantation, deferred kidney transplantation might be the preferred strategy after hepatic allograft engraftment occurred. However, this strategy requires allocation rules where kidney allocation is furthermore prioritized in a scenario of kidney-after-liver transplantation. Regardless of delayed or deferred kidney transplantation, both strategies follow the principle of best donor organ utilization (Figure 1). 
Although SLKT offers an unquestionable survival benefit for many patients with ESLD and simultaneous chronic kidney disease and/or acute kidney injury, the study by the French group highlights the importance of appropriate selection and management of patients undergoing ReLT-KT or SLKT. Future research priorities should focus on contemporary SLKT studies incorporating both renal indications of chronic kidney disease and acute kidney injury in order to identify the highest risk patients and to avoid futile SLKT and ReLT-KT. 


\section{References:}

1. Grant L, Tujios S, Singal AG. Outcomes of simultaneous liver-kidney transplantation: implications for patient selection. Curr Opin Organ Transplant. 2018;23(2):264-270.

2. Miles CD, Westphal S, Liapakis A, et al Simultaneous liver-kidney transplantation: impact on liver transplant patients and the kidney transplant waiting list. Curr Transplant Rep. 2018;5(1):1-6.

3. Cauchy F, Vibert E, Cherqui D, et al. Liver retransplantation associated with kidney transplantation for end stage liver graft disease and renal insufficiency: a morbid procedure on a unique subgroup of patients. Transplantation. IN PRESS

4. Formica RN, Aeder M, Boyle G, et al. Simultaneous liver-kidney allocation policy: a proposal to optimize appropriate utilization of scarce resources. Am J Transplant. 2016;16(3):758-766.

5. Lunsford KE, Bodzin AS, Markovic D, et al. Avoiding futility in simultaneous liver-kidney transplantation: analysis of 331 consecutive patients listed for dual organ replacement. Ann Surg. 2017;265(5):1016-1024.

6. Linecker M, Krones T, Berg T, et al. Potentially inappropriate liver transplantation in the era of the "sickest first" policy - A search for the upper limits. J Hepatol. 2018;68(4):798-813.

7. Ekser B, Mangus RS, Kubal CA, et al. Excellent outcomes in combined liver-kidney transplantation: Impact of kidney donor profile index and delayed kidney transplantation. Liver Transpl. 2018;24(2):222-232. 


\section{Figure legend}

Figure 1: Strategies to avoid futile outcome in combined liver and kidney transplantation.

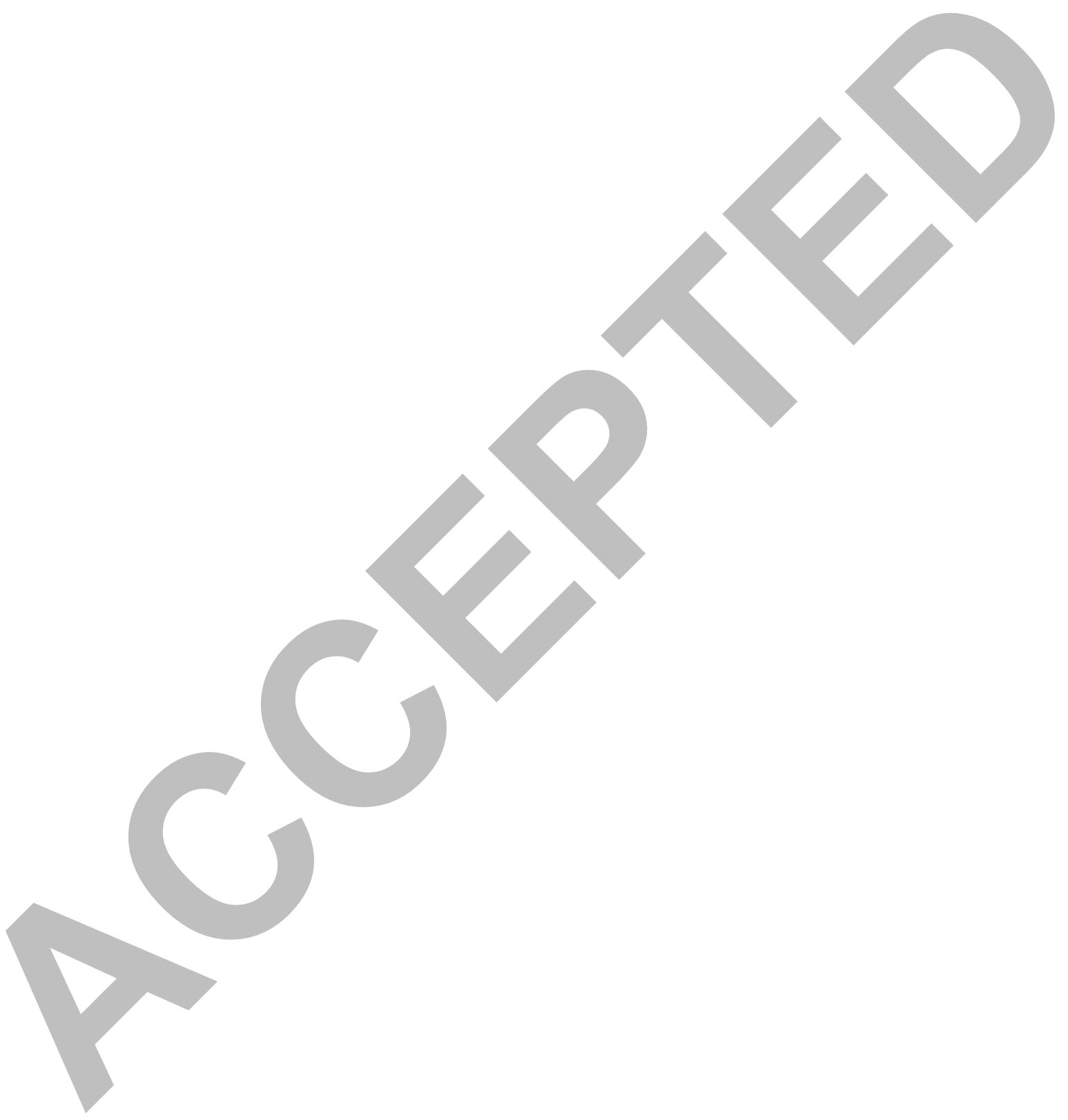


Figure 1

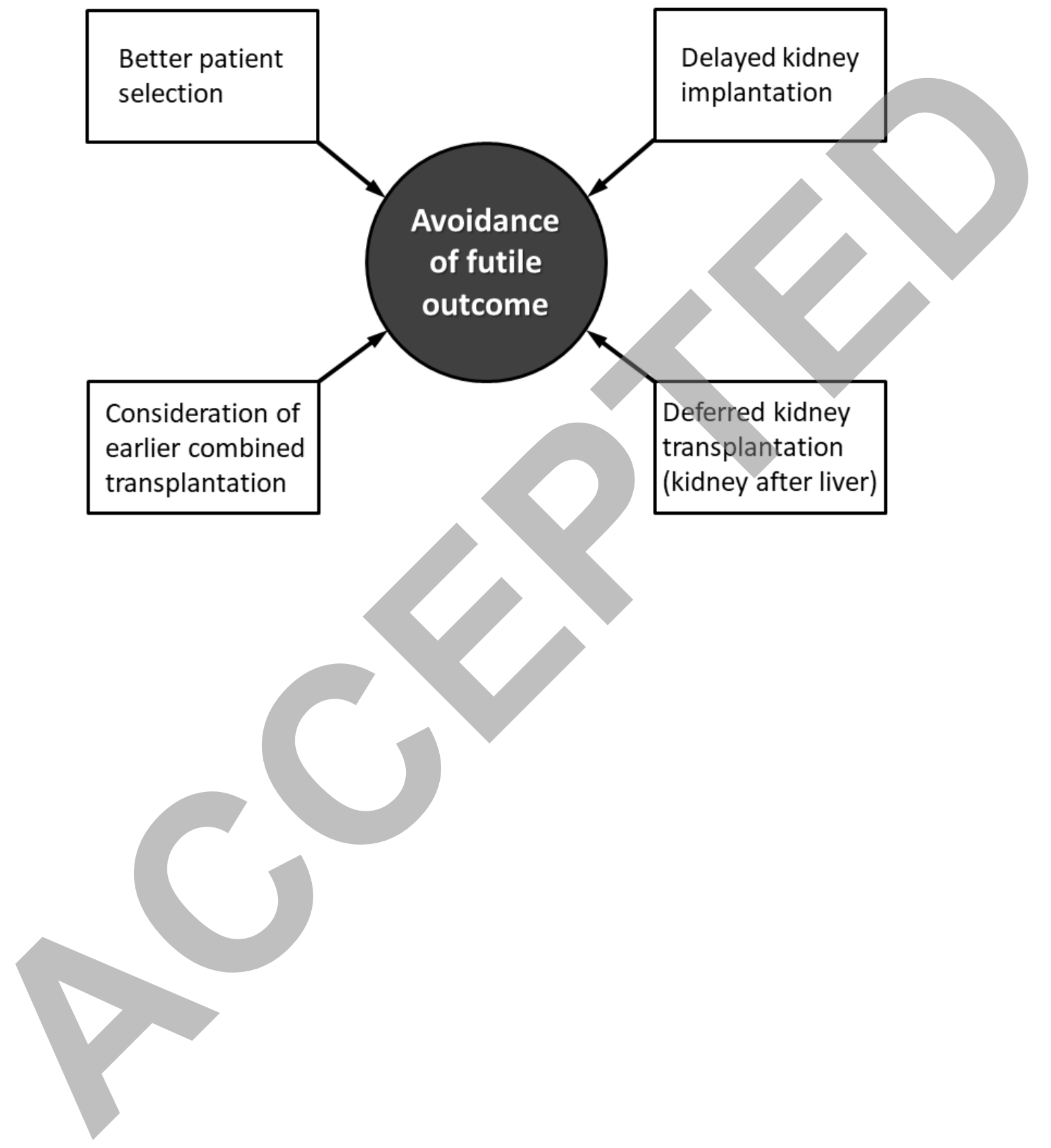

